\title{
Right mini-thoracotomy approach reduces hospital stay and transfusion of mitral or tricuspid valve reoperation with non-inferior efficacy: evidence from propensity-matched study
}

\author{
Qing Wang", Xiaofei Xue\#, Jie Yang", Qian Yang, Pei Wang, Liaoyuan Wang, Peng Zhang, Suyu Wang, \\ Jing Wang, Jibin Xu, Jian Xiao, Zhinong Wang \\ Department of Cardiothoracic Surgery, Changzheng Hospital, Second Military Medical University, Shanghai 200003, China \\ Contributions: (I) Conception and design: Q Wang, X Xue; (II) Administrative support: J Xiao, Z Wang; (III) Provision of study materials or patients: \\ J Xu, Q Yang, P Wang; (IV) Collection and assembly of data: L Wang, J Yang, J Wang; (V) Data analysis and interpretation: P Zhang, S Wang; (VI) \\ Manuscript writing: All authors; (VII) Final approval of manuscript: All authors. \\ \#These authors contributed equally to this work. \\ Correspondence to: Zhinong Wang; Jian Xiao. Department of Cardiothoracic Surgery, Changzheng Hospital, Second Military Medical University, \\ Shanghai 200003, China. Email: wangzn007@163.com; 18916066266@189.cn.
}

Background: There is limited evidence about the efficacy and cost difference between minimally invasive and conventional valve reoperation. This study intended to compare the short-term efficacy and cost between right mini-thoracotomy approach and median sternotomy approach in valve reoperation.

Methods: From Feb 2011 to Sep 2017, 156 patients underwent valve reoperation including 68 cases of minimally invasive approach and 88 cases of traditional median sternotomy approach in our hospital. A propensity scoring was used to match patients with similar demographic characteristics. A total of 42 pairs of patients were left and divided into the conventional sternotomy group (CS group) and the right minithoracotomy group (RT group). A retrospective study of efficacy and cost was conducted between two groups.

Results: There was no statistical difference between two groups in demographical characteristics after propensity-scoring match ( $\mathrm{P}>0.05)$. In-hospital mortality was $11.9 \%(5 / 42)$ for CS group and 7.1\% (3/42) for the RT group $(\mathrm{P}=0.687)$. No significant disparity was found in the incidence of complications between two groups $(\mathrm{P}>0.05)$. $\mathrm{CPB}$ time $(\mathrm{P}=0.012)$, bypass time $(\mathrm{P}=0.006)$ and operation time $(\mathrm{P}=0.003)$ of $\mathrm{CS}$ group were significantly higher than RT group. Blood loss $(\mathrm{P}=0.014)$ and transfusion volume $(\mathrm{P}=0.003)$ of RT group was less than CS group. Shorter ICU and hospital stay was seen in RT group compared with CS group $(\mathrm{P}<0.001)$. Though the materials cost of RT group was higher than CS group $(\mathrm{P}<0.001)$, no significant disparity was found in total cost between CS group and RT group $(\mathrm{P}=0.790)$.

Conclusions: The right mini-thoracotomy approach can achieve equivalent efficacy with conventional median approach, and doesn't necessarily increase the total cost. Moreover, the minimally invasive approach can decrease the operation time, hospital stay and blood product transfusion.

Keywords: Valve reoperation; right mini-thoracotomy; sternotomy; cost analysis; propensity score match

Submitted Mar 02, 2018. Accepted for publication Jun 06, 2018.

doi: $10.21037 /$ jtd.2018.07.53

View this article at: http://dx.doi.org/10.21037/jtd.2018.07.53 


\section{Introduction}

With the popularity of bioprosthetic valves and the elongation of patient longevity, valve reoperation becomes more and more common in most cardiac surgery centers (1). However, valve reoperation is also quite challenging due to the difficulty in exposure of the incision. Conventional redo valve surgery routinely adopts the sternotomy as the incision, which may be highly risky in injuring the heart and great vessels (2). Compared with patients receiving initial valve operation, those receiving redo operations have significantly higher mortality and morbidity (3).

Tremendous evidence has proven the efficacy and advantages of minimally invasive cardiac surgery over conventional operations (4). Right mini-thoracotomy is the most common incision of minimally invasive mitral or tricuspid valve surgery, which is also reported in mitral reoperation (5). Although some retrospective studies have demonstrated that minimally invasive approach is useful and available in reoperations, there is limited comparison study between minimally invasive approach and conventional sternotomy approach. In this study, we compared two approaches in operative data, short-term efficacy, as well as detailed cost.

\section{Methods}

\section{Study population}

From Feb 2011 to Sep 2017, a total of 160 adult patients received redo mitral or tricuspid operations in Changzheng Hospital affiliated to Second Military Medical University in China. To minimize the confounding bias, we excluded patients meeting the following criteria: patients under the age of 18 years old; patients in critical conditions; patients whose previous chest operation was not cardiac surgery; patients undergoing greater than or equal to third time redo. A total of 144 patients were enrolled in the study. Patient data and detailed hospital cost was collected from each individual and analyzed retrospectively. Propensity score of 144 patients were calculated using the logistic regression model based on demographical variables, and the patients with nearest scores were matched and enrolled in this study. In the end, there were 42 pairs of patients left for further study. Patient enrolled were classified into the right mini-thoracotomy group (RT group) and conventional sternotomy group (CS group) according to their approach. The study design was shown as flow chart of Figure 1.

The anonymous study was approved by the Committee on Ethics of Biomedicines, Second Military Medical University, with the patient consent not required. All data was collected from the record system of Changzheng Hospital, with all personal data secured.

\section{Surgical techniques}

All patients ready to receive redo operations would be given CT scan preoperatively, with a group discussion made to decide the final approach according to the clinical characteristics of the patient. If there are any contraindications of minimally invasive approach, such as severe intra-pleural adhesions, severe obesity or chest wall deformities, the traditional sternotomy would be the choice and vice versa. If both approach works, the finical decision would be made by the patient and the surgeon together.

Patient receiving right mini-thoracotomy were intubated with double-lumen endotracheal tubes. SwanGanz catheters were used in every patient, and the fourth intercostal space was predominantly chosen as the incision point, with femoral cannulation completed following the thoracotomy to initiate the cardiopulmonary bypass (CPB). Standardized procedures of mitral valve (MV) replacement or repair, and tricuspid valve repair or replacement were conducted accordingly. Transthoracic clamping (using a Chitwood clamp) in the right axilla was used to accomplish the aortic occlusion, and either antegrade or retrograde cold blood cardioplegia was used for myocardial protection. The left atrium was entered into along the interatrial groove or through the atrial septum in cases in which tricuspid problems were present. Routine procedures including deairing and transesophageal echocardiography study were also completed as part of this study.

Patients receiving conventional sternotomy approach were intubated with single lumen endotracheal tubes, and hemodynamics was monitored by swan-Ganz catheters. Following median careful re-sternotomy with swing saw, aorto-bicaval cannulation was conducted to establish CPB. Standardized procedures of MV replacement or repair, and tricuspid valve repair or replacement were performed likewise.

\section{Data collection}

All data was collected from the electronic medical record system of the hospital and the record of CPB group. Demographical characteristics including gender, age and BMI, clinical characteristics including cardiac function, Log Euroscore, Parsonnet score, cause of surgery, 

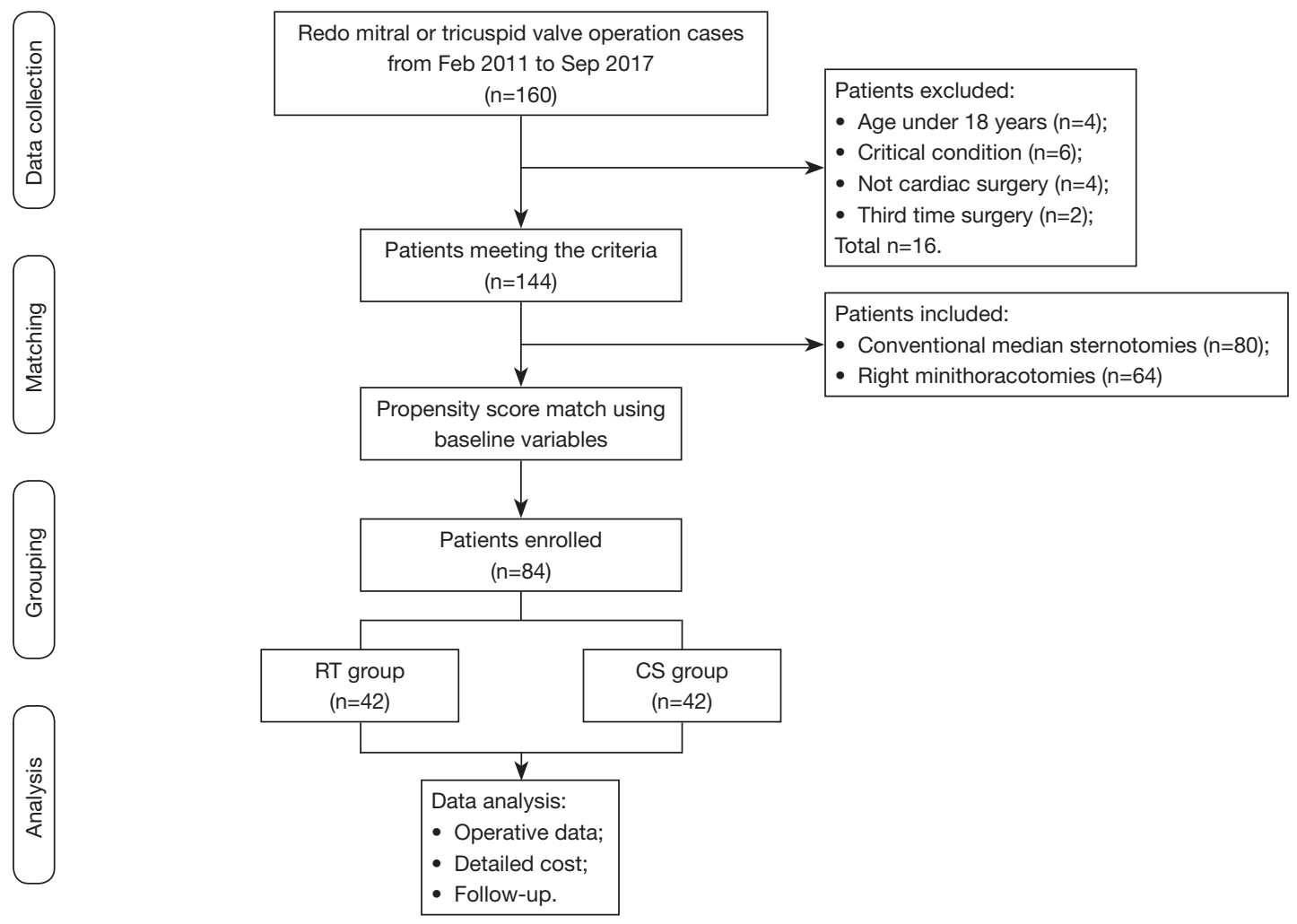

Figure 1 Flow chart of study design.

echocardiography, comorbidities, previous cardiac surgery, surgical type were collected. We also collected postoperative information including in-hospital death, time to discharge, time of ICU stay, blood loss, chest tube drainage, transfusion amount, CPB data, and complications. Cost information was also included in the analysis.

\section{Follow-up}

All patients were required to complete serial clinical follow-up. Transthoracic echocardiography, electrocardiogram (ECGs) and chest $\mathrm{X}$-rays were used to evaluate cardiac function and the condition of the MV and tricuspid valve. At first year after discharge, the follow-up in out-patient clinics was done every three months. After first year, annual examine in clinics was conducted. If the patients were readmitted to the hospital during follow-up, the reason of admission were recorded.

\section{Statistical analysis}

IBM SPSS Statistics, version 19.0 (SPSS, Inc, Armonk,
NY, USA) was used for statistical analysis. Continuous variables are presented as mean \pm standard deviations and categorical variables are presented as proportions. Student's $t$-test and $\chi^{2}$ test were performed in different evaluations of continuous variables and categorical variables separately, prior to matching.

Multivariable logistic regression was used to calculate the propensity score of all patients by taking into account perioperative variables. Patients with closest propensity scores were matched using the "greedy match" method. Following the propensity score match, a Student's t-test of paired samples and a McNemar test were adopted in the analysis.

\section{Results}

\section{Demographical and clinical characteristics}

The demographical and clinical characteristics of the patients before and after the propensity score match are shown in Tables 1,2. Before the propensity score 
Table 1 Demographical and clinical characteristics of overall patients undergoing valve reoperation

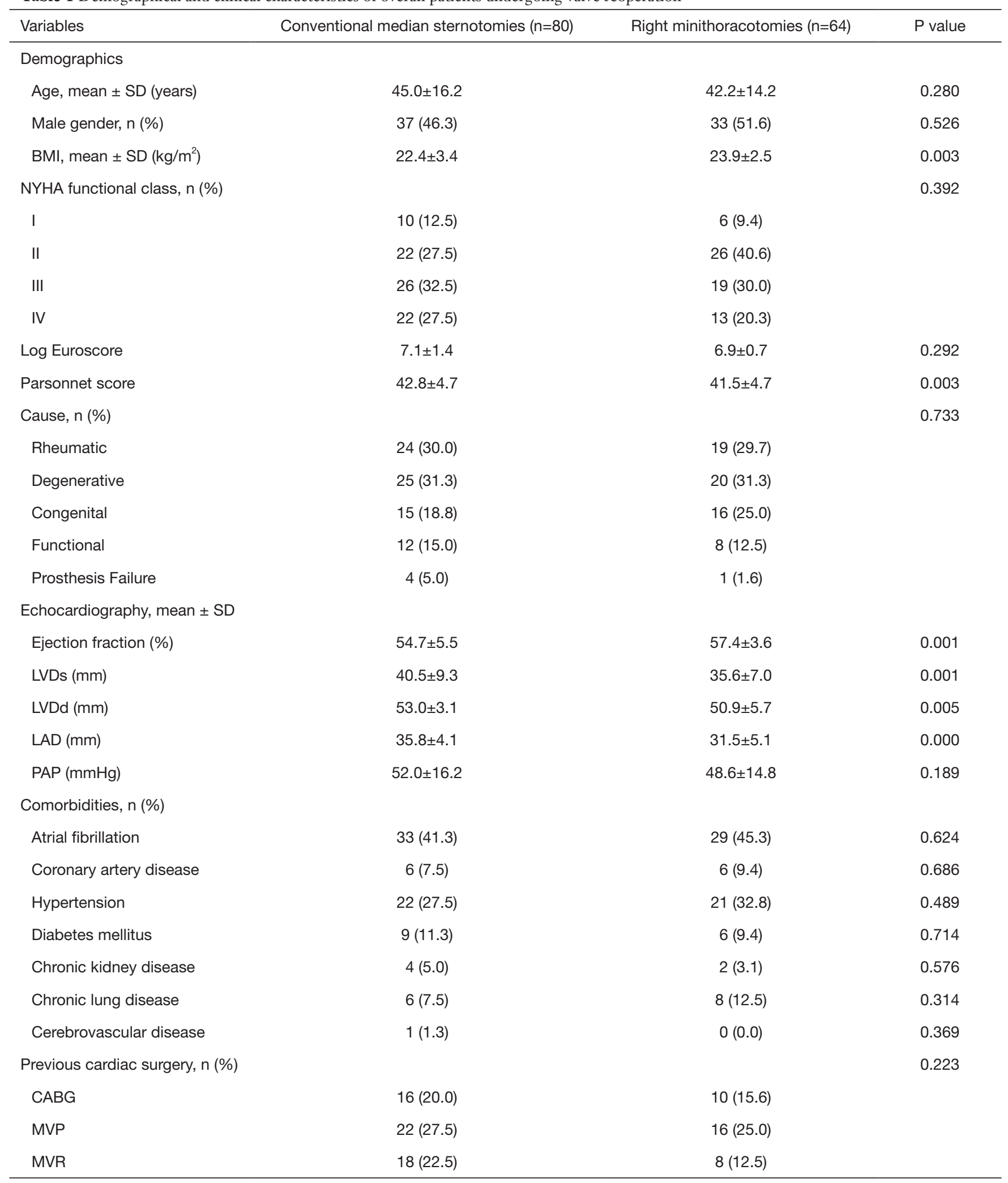

Table 1 (continued) 
Table 1 (continued)

\begin{tabular}{lcc}
\hline Variables & Conventional median sternotomies $(\mathrm{n}=80)$ & Right minithoracotomies $(\mathrm{n}=64)$ \\
\hline AVR & $9(11.3)$ & $15(23.4)$ \\
Congenital cardiac surgery & $14(17.5)$ & $12(18.8)$ \\
Atrial myxoma & $2(2.5)$ & $3(4.7)$ \\
Contemporary surgery, $\mathrm{n}(\%)$ & & $20(31.3)$ \\
MVR or MVP & $26(32.5)$ & $26(40.6)$ \\
TVR or TVP & $15(18.8)$ & $18(28.1)$ \\
MVR/MVP + TVP & $39(48.8)$ & 0.007 \\
\hline
\end{tabular}

BMI, body mass index; NYHA, New York Heart Association; TR, tricuspid regurgitation; LVDs, left ventricular diameter systolic; LVDd, left ventricular diameter diastolic; LAD, left atrial diameter; PAP, pulmonary artery pressure; CABG, coronary artery bypass graft; MVR, mitral valve replacement; MVP, mitral valve repair; TVR, tricuspid valve replacement; TVP, tricuspid valve repair.

Table 2 Demographical and clinical characteristics of propensity-scoring matched patients undergoing valve reoperation

\begin{tabular}{|c|c|c|c|}
\hline Variables & CS group $(n=42)$ & RT group $(n=42)$ & $P$ value \\
\hline Age, mean \pm SD (years) & $44.24 \pm 18.90$ & $43.64 \pm 15.38$ & 0.874 \\
\hline Male gender, n (\%) & $18(42.9)$ & $20(47.6)$ & 0.815 \\
\hline $\mathrm{BMI}$, mean $\pm \mathrm{SD}\left(\mathrm{kg} / \mathrm{m}^{2}\right)$ & $22.91 \pm 2.77$ & $23.13 \pm 3.44$ & 0.751 \\
\hline I & $4(9.5)$ & $4(9.5)$ & \\
\hline II & $12(28.6)$ & $10(23.8)$ & \\
\hline III & $12(28.6)$ & $16(38.1)$ & \\
\hline IV & $14(33.3)$ & $12(28.6)$ & \\
\hline Cause, n (\%) & & & 0.174 \\
\hline Rheumatic & $14(33.3)$ & $10(23.8)$ & \\
\hline Degenerative & $12(28.6)$ & $10(23.8)$ & \\
\hline Congenital & $8(19.0)$ & $12(28.6)$ & \\
\hline Functional & $6(14.3)$ & $4(9.5)$ & \\
\hline Prosthesis Failure & $2(4.8)$ & $6(14.3)$ & \\
\hline \multicolumn{4}{|l|}{ Echocardiography, mean \pm SD } \\
\hline Ejection fraction (\%) & $56.45 \pm 4.48$ & $56.26 \pm 3.97$ & 0.837 \\
\hline
\end{tabular}

Table 2 (continued) 
Table 2 (continued)

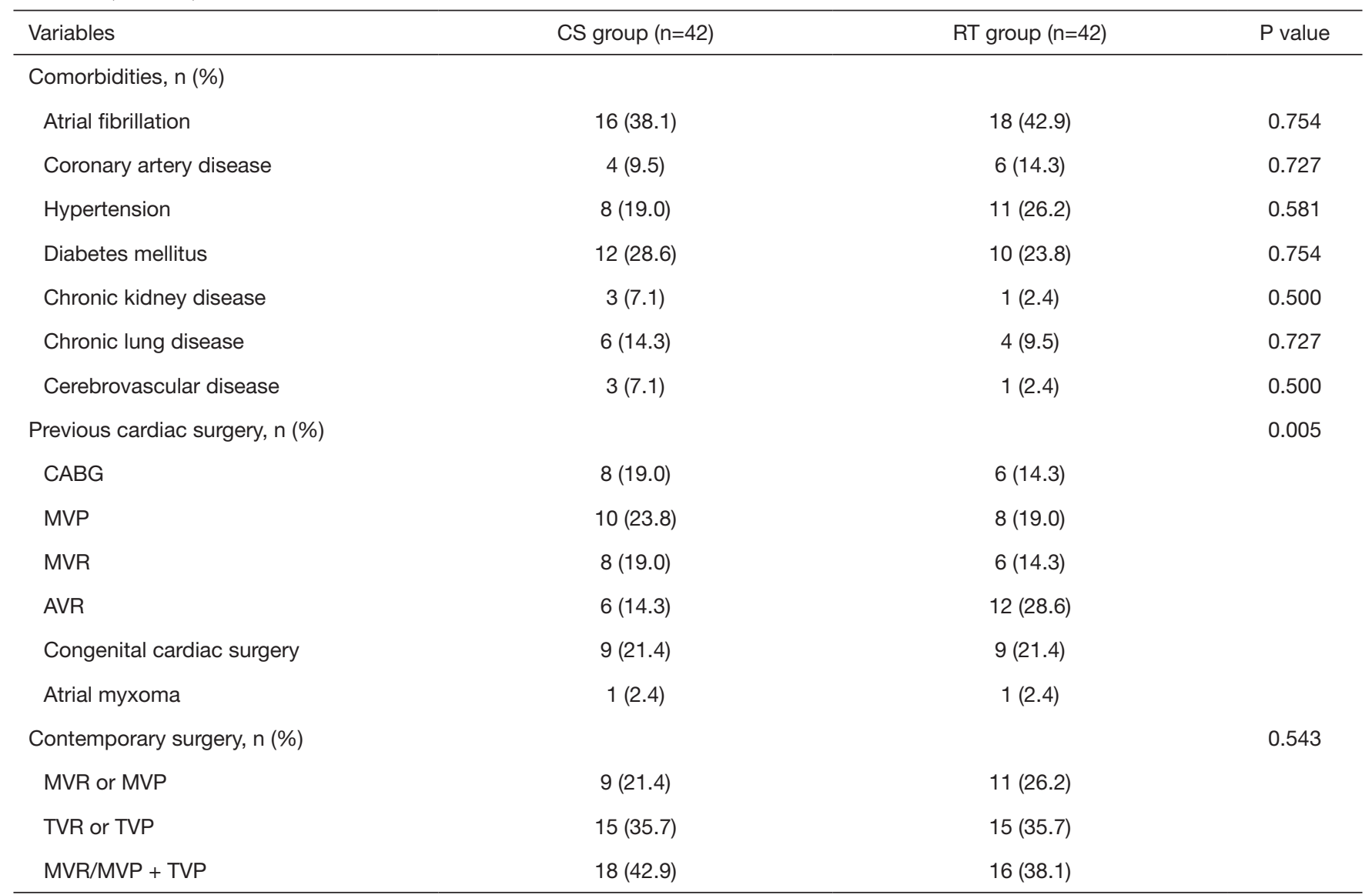

BMI, Body mass index; NYHA, New York Heart Association; TR, tricuspid regurgitation; LVDs, Left ventricular diameter systolic; LVDd, Left ventricular diameter diastolic; LAD, Left atrial diameter; PAP, Pulmonary artery pressure; CABG, Coronary artery bypass graft; MVR, Mitral valve replacement; MVP, Mitral valve repair; TVR, Tricuspid valve replacement; TVP, Tricuspid valve repair

match, there was a significant difference between two groups in $\mathrm{BMI}(\mathrm{P}=0.003)$, Parsonnet score $(\mathrm{P}=0.003)$, echocardiography results $(\mathrm{P}=0.001)$, and the type of contemporary surgery $(\mathrm{P}=0.007)$. After the match, there was no statistical difference between two groups $(\mathrm{P}>0.05)$, with the exception of previous cardiac surgery $(\mathrm{P}=0.005)$. There were six types of pervious cardiac surgery, with the paired sample statistics adopted, making the match difficult. However, when independent sample chi-square was used rather than paired sample, there would be no significant difference between two groups $(\mathrm{P}=0.732)$.

\section{Perioperative data and in-hospital outcomes}

As was shown in Table 3, there was no difference between
CS group and RT group in in-hospital death $(11.9 \%$ vs. $7.1 \%, \mathrm{P}=0.687)$. Five patients of CS group died in hospital, when two died of severe pneumonia, one died of acute respiratory distress syndrome (ARDS), one died of low cardiac output syndrome, one died of major bleeding. Three patient of RT group died in hospital, while one patient died because of sepsis and two died of severe pneumonia. The extubation time didn't differ between two groups $(\mathrm{P}=0.479)$, while the ICU stay and the time to discharge of RT group were significantly less than those of CS group $(\mathrm{P}<0.001)$. As to the chest tube drainage, the amount of RT group was significantly lower than that of CS group $(\mathrm{P}<0.001)$. Also, the operative blood loss was much less in RT group $(\mathrm{P}=0.014)$.

As a result, the transfusion categories and amount were 
Table 3 Perioperative data and in-hospital outcomes of propensity-matched groups

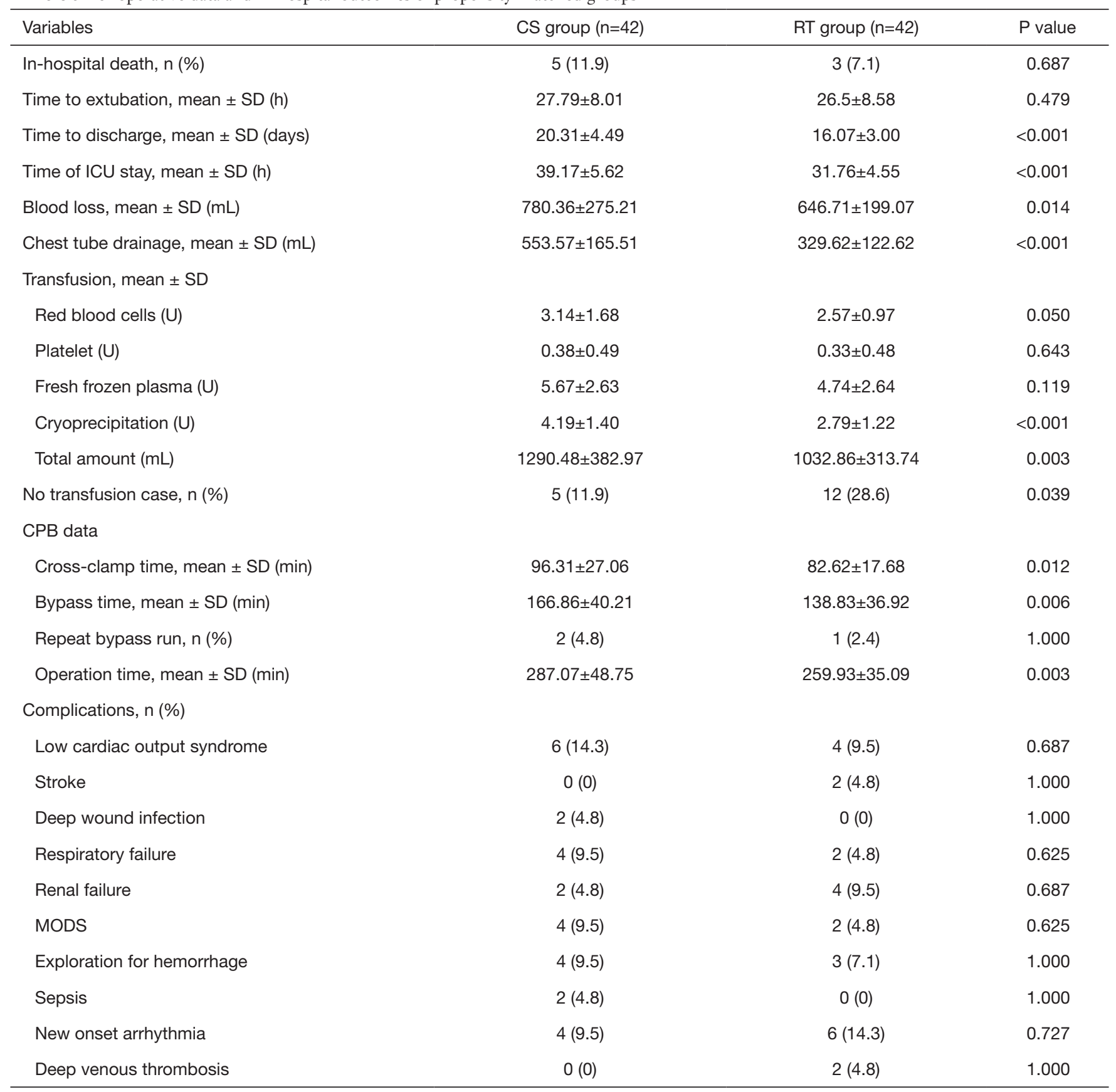

CPB, cardiopulmonary bypass; TVP, tricuspid valve repair; MODS, multiple organ dysfunction syndrome.

different between two groups, the CS group consumed more red blood cells $(\mathrm{P}=0.050)$ and cryoprecipitation $(\mathrm{P}<0.001)$, with no statistical disparity in platelet $(\mathrm{P}=0.643)$ and fresh frozen plasma $(\mathrm{P}=0.119)$, and the total amount of transfusion is much higher for CS group $(\mathrm{P}=0.003)$. The operative data including cross-clamp time $(\mathrm{P}=0.012)$, bypass time $(\mathrm{P}=0.006)$, and operation time $(\mathrm{P}=0.003)$ were significantly less for RT group, while the incidence of postoperative complications was close between two groups (all $\mathrm{P}>0.05$ ). The comparison of postoperative complications was shown in Figure 2. We should notice that there were two cases of stroke in RT group, with none in 


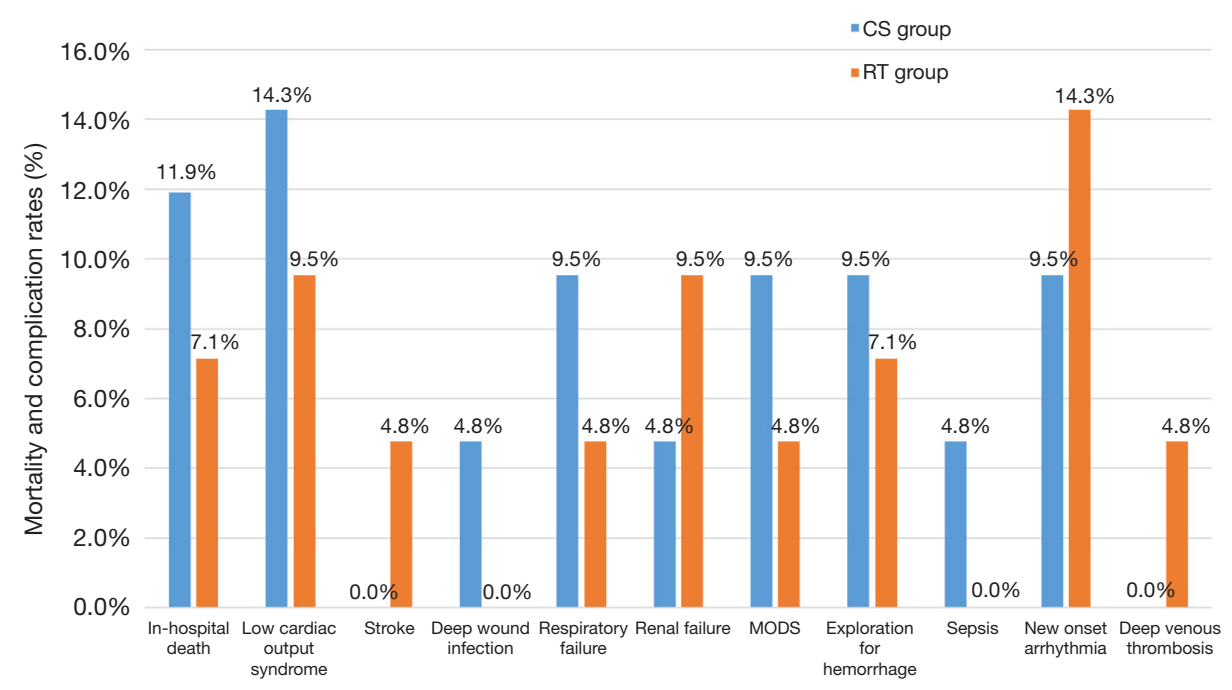

Figure 2 In-hospital death and complications of propensity-matched groups. CS group, conventional sternotomy group; RT group, right mini-thoracotomy group.

CS group ( $\mathrm{P}=1.000)$, the two patients were transferred to rehabilitation hospital after the acute phase. There were no complications led by femoral cannulation happened in RT group such as lower leg ischemia, retrograde aortic dissection, re-expansion pulmonary edema.

\section{Cost analysis}

The detailed cost of CS group and RT group were analyzed, when all insurance data were excluded, as well as abnormal values of dead cases. We categorized the cost into direct cost and indirect cost, as was illustrated in Table 4 and Figure 3. Patients in CS group spent more money on direct cost $(\mathrm{P}=0.034)$. Although patients of RT group spent more on material cost $(\mathrm{P}<0.001)$, they saved money on other items of indirect cost, such as boarding, nursing and food because of shorter hospital stay $(\mathrm{P}<0.001)$. In consequence, there was no difference between two groups in total cost $(\mathrm{P}=0.790)$.

\section{Follow-up}

As was shown in Table 5, follow-up was completed (100\%) with a mean duration of $3.4 \pm 1.4$ years for CS group and $3.5 \pm 1.7$ years for RT group. During the follow-up, a total of 35 patients were admitted into hospital again for following reasons including reoperation, heart failure, arrhythmia, anemia, and pleural effusion. There was no difference in readmission rate between two groups $(\mathrm{P}=0.648)$.

\section{Discussion}

Recent years have witnessed a significant increase of redo valve surgery in cardiac centers for the popularity of bioprosthesis and the elongation of lifespan (6). There were there patients in this study received the redo operation due to bioprosthesis failure, while other patients were confronted with the valve problem again. Although the inhospital mortality of reoperation has reduced significantly because of the myocardial protection improvement, new CPB strategies, and the appropriate approach, it is still challenging and risky for most cardiac surgeons (2). Major problem led by reoperation is the re-exposure of the heart, mostly attached to the sternum due to the extensive pericardial adhesions, which may contribute to the injury of cardiac structures. Right mini-thoracotomy provides another approach to conduct mitral or tricuspid valve surgery, which has been reported to achieve satisfying short and long term results compared with conventional sternotomy approach (7). Several retrospective studies have reported the availability and usefulness of right minithoracotomy in mitral or tricuspid valve reoperation (5,8-15). However, these studies have unavoidable disadvantages such as small sample size, no control group. A study demonstrates that re-sternotomy is also safe with zero major cardiac 
Table 4 Total hospital cost stratified by billing category (US dollars)

\begin{tabular}{|c|c|c|c|}
\hline Cost category & CS group $(n=42)$ & $\mathrm{RT}$ group $(\mathrm{n}=42)$ & $P$ value \\
\hline Pharmacy & $5,002.7 \pm 551.0$ & $4,679.7 \pm 450.5$ & 0.005 \\
\hline Laboratory test & $1,062.9 \pm 123.5$ & $1,047.0 \pm 143.0$ & 0.579 \\
\hline Imaging & $607.0 \pm 49.4$ & $793.5 \pm 140.2$ & $<0.001$ \\
\hline Operation & $1,157.8 \pm 331.0$ & $1,218.4 \pm 342.7$ & 0.449 \\
\hline Anesthesia & $683.2 \pm 165.1$ & $504.4 \pm 94.6$ & $<0.001$ \\
\hline Blood bank & $1,225.4 \pm 217.8$ & $1,117.3 \pm 203.7$ & 0.024 \\
\hline Indirect cost (\$) & $11,653.7 \pm 1702.4$ & $12,159.0 \pm 1093.7$ & 0.080 \\
\hline Nursing & $150.3 \pm 33.8$ & $97.0 \pm 12.9$ & $<0.001$ \\
\hline Food & $101.1 \pm 15.9$ & $86.7 \pm 14.0$ & $<0.001$ \\
\hline Others & $9,011.0 \pm 1679.4$ & $8,400.0 \pm 1124.8$ & 0.046 \\
\hline Total (\$) & $23,049.7 \pm 1783.2$ & $23,136.0 \pm 1383.7$ & 0.790 \\
\hline
\end{tabular}

CS group, conventional sternotomy group; RT group, right mini-thoracotomy group.

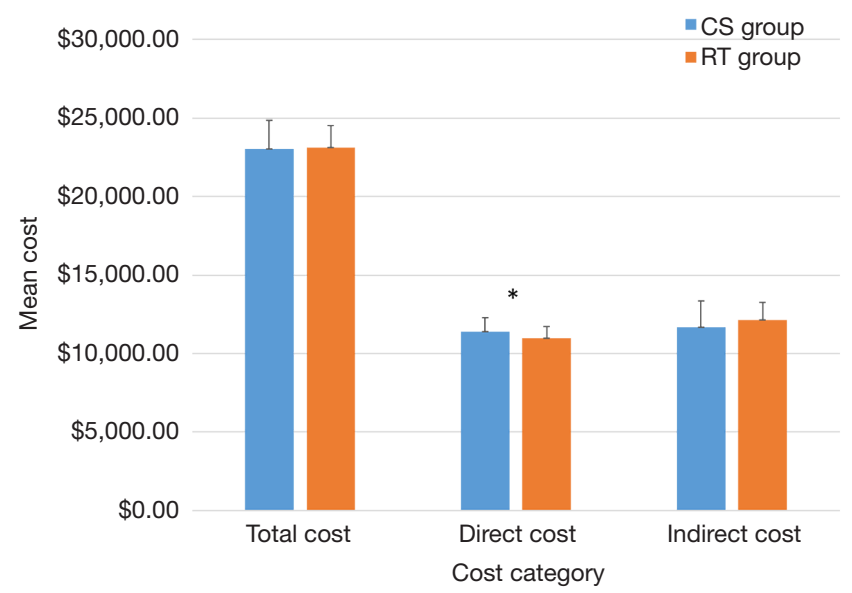

Figure 3 Total and stratified hospital cost of propensity-matched groups. CS group, conventional sternotomy group; RT group, right mini-thoracotomy group. ${ }^{*}, \mathrm{P}<0.05$.

injury/catastrophic hemorrhage during reoperation (16). In addition, there is some controversies about the mortality, transfusion amount, and the incidence of perioperative complications between two approaches. Hence, we conduct the retrospective propensity-score matched study to provide
Table 5 Follow-up results after discharge

\begin{tabular}{lccc}
\hline Variables & CS group $(n=42)$ & RT group $(n=42)$ & P value \\
\hline Follow-up years & $3.4 \pm 1.4$ & $3.5 \pm 1.7$ & 0.921 \\
Readmission & $16(38.1)$ & $19(45.2)$ & 0.648 \\
Reoperation & $2(4.8)$ & $2(4.8)$ & \\
Heart failure & $4(9.5)$ & $6(14.3)$ & \\
Arrhythmia & $8(19.0)$ & $7(16.7)$ & \\
Anemia & $2(4.8)$ & $2(4.8)$ & \\
Pleural effusion & $0(0)$ & $2(4.8)$ &
\end{tabular}

CS group, conventional sternotomy group; RT group, right minithoracotomy group.

more evidence.

Preoperative chest computed tomography (CT) scan must be performed on all patients receiving redo valve surgery in our center to identify the relationship between the sternum and important structures. Also, previous rightsided thoracotomy or severe adhesions must be evaluated before adopting the right mini-thoracotomy approach. Inhospital mortality of mitral reoperation varies between 
different studies. Onnasch et al. reported a mortality of $5.1 \%$ of 39 patients undergoing MV repair or replacement via a right minithoracotomy (8). Seeburger et al. summarize the results of 181 consecutive patients receiving minimally invasive redo MV surgery, and 30-day mortality was $6.6 \%$ (5). However, these authors didn't report the mortality of patients of sternotomy. We compared the mortality of two groups, and the mortality is $11.9 \%$ for CS group and $7.1 \%$ for RT group, with no statistical difference between two groups. In this study, the mortality rate is higher than previous Euroscore estimates. What's more, pervious study has demonstrated an overestimated risk of $\log$ euro for cardiac surgery patients (17). However, we believe there are two major reasons led to the relative higher mortality than preoperative expectation. First of all, the study of Euroscore only explored the patients undergoing coronary artery bypass graft rather than valve operation, and a subgroup analysis of redo-operation wasn't conducted too, which make the conclusion not applicable for redo situation. Second, the sample size of this study is relatively small, hence the comprehensive mortality might not be correlated with the Euroscore in general. Our results also showed that the perioperative blood loss was significantly less in RT group, which in turn mitigated the requirement of transfusion. All types of blood product were saved in RT group, with a significant difference in red blood cells and cryoprecipitation. Hanedan et al. reported 30 cases of redo tricuspid replacement, with a median re-sternotomy in 13 patients and a right antero-lateral thoracotomy in 17 patients. His results showed that the postoperative drainage amounts were lower in the thoracotomy group (15). The right mini-thoracotomy resulted in less blood loss mainly for its shorter incision and less chance of major bleeding. As previous reported, minimally invasive cardiac surgery gains advantages of faster recovery, shorter hospital (9), and our study also showed that the hospital stay and ICU stay of RT group was shorter than the CS group, which could be attributed to minimized incision, shortened $\mathrm{CPB}$ time and operation time. However, several studies and our previous study focusing on MV repair have reported that the mini-incision can increase the operation time and CPB time (18-21). However, with the growing experience in femoral cannulation and surgical technique of mini-incision, the operation time and bypass time was obviously decreased compared with traditional sternotomy, which was burdened with great trouble in detaching and cannulation. There are previous reports of higher stroke rates (22), complications led by femoral cannulation, such as lower limb ischemia (23), retrograde aortic dissection (22), as well as re-expansion pulmonary edema (24) led by single ventilation for right mini-thoracotomy approach. However, no difference of stroke incidence and other special complications were observed in this study, which could be attributed to perioperative anti-coagulation therapy and advancing techniques. A short-term followup in our study demonstrated that there was no difference between two groups in readmission rates.

Although the minimally invasive cardiac surgery has been promoted for years, it is still facing some obstacles in China, and one of the major reasons is the economic reason. Materials such as femoral cannula and additional imaging test adds about $¥ 10,000$ to the total cost. However, our study showed that there was no difference between two groups in total cost, because the RT group saved the equal money in direct cost such as pharmacy, treatment and blood transfusion.

Some limitations of this study cannot be fully avoided. First, due to the relatively small population undergoing redo cardiac surgery, our sample size is not powerful enough to find some important difference such as the complication of stroke. Second, a retrospective study cannot fully eliminate the subject factors which may affect the results even though a propensity score match was conducted. Last, due to different insurance policy and materials price, the cost may differ a lot in different places. Therefore, our study only provides some new perspectives in the cost of reoperations.

\section{Conclusions}

In comparison with conventional sternotomy, the right mini-thoracotomy can achieve non-inferior efficacy, reduce the operation time, blood transfusion and shorten hospital stay without increasing the total cost in redo valve surgery. Therefore, we can conclude that minimally invasive approach is an effective and advantageous alternative for most mitral or tricuspid valve reoperation.

\section{Acknowledgements}

Funding: This study was funded by the National Nature Science Foundation of China (No. 81670299), Shanghai Shenkang Emerging Advanced Technology Project (No. 
SHDC12014107), Leading Medicine Project of Shanghai Science and Technology Committee (No. 15411960100).

\section{Footnote}

Conflicts of Interest: The authors have no conflicts of interest to declare.

Ethical Statement: The anonymous study was approved by the Committee on Ethics of Biomedicines, Second Military Medical University, with the patient consent not required.

\section{References}

1. Maganti M, Rao V, Armstrong S, et al. Redo Valvular Surgery in Elderly Patients. Ann Thorac Surg 2009;87:521-5.

2. Furukawa H, Tanemoto K. Redo Valve Surgery-Current Status and Future Perspectives. Ann Thorac Cardiovasc Surg 2014;20:267-75.

3. Tyers GF, Jamieson WR, Munro AI, et al. Reoperation in biological and mechanical valve populations: fate of the reoperative patient. Ann Thorac Surg 1995;60:S464-8; discussion S468-9.

4. Cohn LH, Adams DH, Couper GS, et al. Minimally invasive cardiac valve surgery improves patient satisfaction while reducing costs of cardiac valve replacement and repair. Ann Surg 1997;226:421-6.

5. Seeburger J, Borger MA, Falk V, et al. Minimally invasive mitral valve surgery after previous sternotomy: experience in 181 patients. Ann Thorac Surg 2009;87:709-14.

6. Adams DH, Filsoufi F, Byrne JG, et al. Mitral valve repair in redo cardiac surgery. J Card Surg 2002;17:40-5.

7. McClure RS, Athanasopoulos LV, McGurk S, et al. One thousand minimally invasive mitral valve operations: Early outcomes, late outcomes, and echocardiographic follow-up. J Thorac Cardiovasc Surg 2013;145:1199-206.

8. Onnasch JF, Schneider F, Falk V, et al. Minimally invasive approach for redo mitral valve surgery: a true benefit for the patient. J Card Surg 2002;17:14-9.

9. Bolotin G, Kypson AP, Reade CC, et al. Should a videoassisted mini-thoracotomy be the approach of choice for reoperative mitral valve surgery? J Heart Valve Dis 2004;13:155-8; discussion 158.

10. Casselman FP, La Meir M, Jeanmart H, et al.
Endoscopic mitral and tricuspid valve surgery after previous cardiac surgery. Circulation 2007;116:I270-5.

11. Svensson LG, Gillinov AM, Blackstone EH, et al. Does right thoracotomy increase the risk of mitral valve reoperation? J Thorac Cardiovasc Surg 2007;134:677-82.

12. Kitamura T, Stuklis RG, Edwards J. Redo mitral valve operation via right minithoracotomy--"no touch" technique. Int Heart J 2011;52:107-9.

13. Seo H, Miyawaki I, Higashibeppu N, et al. Investigation of the outcomes and complications in right thoracotomy approach for mitral valve reoperation. Masui 2012;61:1058-63.

14. Botta L, Cannata A, Bruschi G, et al. Minimally invasive approach for redo mitral valve surgery. J Thorac Dis 2013;5:S686-93.

15. Hanedan MO, Cicekcioglu F, Aksoyek A, et al. Tricuspid Valve Replacement Through Right Thoracotomy has Better Outcomes in Redo Cases. Heart Lung Circ 2017;26:88-93.

16. O'Brien MF, Harrocks S, Clarke A, et al. How to do safe sternal reentry and the risk factors of redo cardiac surgery: A 21-year review with zero major cardiac injury. J Card Surg 2002;17:4-13.

17. Karabulut H, Toraman F, Alhan C, et al. EuroSCORE overestimates the cardiac operative risk. Cardiovasc Surg 2003;11:295-8.

18. Wang Q, Xi W, Gao Y, et al. Short-term outcomes of minimally invasive mitral valve repair: a propensitymatched comparison. Interact Cardiovasc Thorac Surg 2018;26:805-12.

19. Jung JC, Kim KH. Minimally Invasive Cardiac Surgery versus Conventional Median Sternotomy for Atrial Septal Defect Closure. Korean J Thorac Cardiovasc Surg 2016;49:421-6.

20. Iribarne A, Easterwood R, Russo MJ, et al. Longterm outcomes with a minimally invasive approach for resection of cardiac masses. Ann Thorac Surg 2010;90:1251-5.

21. Chaney MA, Durazo-Arvizu RA, Fluder EM, et al. Port-access minimally invasive cardiac surgery increases surgical complexity, increases operating room time, and facilitates early postoperative hospital discharge. Anesthesiology 2000;92:1637-45.

22. Modi P, Chitwood WR Jr. Retrograde femoral arterial perfusion and stroke risk during minimally invasive mitral valve surgery: is there cause for concern? Ann Cardiothorac Surg 2013;2:E1. 
23. Dogan S, Aybek T, Risteski PS, et al. Minimally invasive port access versus conventional mitral valve surgery: prospective randomized study. Ann Thorac Surg 2005;79:492-8.

Cite this article as: Wang Q, Xue X, Yang J, Yang Q, Wang P, Wang L, Zhang P, Wang S, Wang J, Xu J, Xiao J, Wang Z. Right mini-thoracotomy approach reduces hospital stay and transfusion of mitral or tricuspid valve reoperation with non-inferior efficacy: evidence from propensity-matched study. J Thorac Dis 2018;10(8):4789-4800. doi: 10.21037/ jtd.2018.07.53
24. Sugiyama Y, Shimizu F, Shimizu S, et al. Severe Reexpansion Pulmonary Edema Induced by One-Lung Ventilation. Respir Care 2015;60:e134-40. 